\title{
Satisfación del estudiante de eduación superior. Caso instituto tecnológico inan extensión girón
}

\section{Higher education student satisfaction. Case: inan technological institute, giron extensión}

DOI: $10.46932 / \mathrm{sfjdv2n2-013}$

Received in: january 1st, 2020

Accepted in: March 30th, 2020

Ramón Edecio Pineda Varela

Magíster en Educación1

Institución: Instituto Tecnológico Integración Andina INAN.

Dirección: Mariscal Lamar 18-77 y Abraham Sarmiento, Cuenca - Azuay - Ecuador

E-mail: elcesar1987@hotmail.com

\section{Mariela Fernanda Ortega Riascos}

Economista

Institución: Instituto Tecnológico Integración Andina INAN.

Dirección: Mariscal Lamar 18-77 y Abraham Sarmiento, Cuenca - Azuay - Ecuador

E-mail: marielaortega886@gmail.com

\section{Elizabeth Alexandra Girón Pastuizaca}

Ingeniera en Contabilidad y Auditoria

Institución: Instituto Tecnológico Integración Andina INAN.

Dirección: Mariscal Lamar 18-77 y Abraham Sarmiento, Cuenca - Azuay - Ecuador

E-mail:alexinan1984@gmail.com

\section{César Gabriel Perero Robles}

Ingeniero en Contabilidad y Auditoría

Institución: Instituto Tecnológico Integración Andina INAN.

Dirección: Mariscal Lamar 18-77 y Abraham Sarmiento, Cuenca - Azuay - Ecuador E-mail: elcesar1987@ hotmail.com

\section{RESUMEN}

El presente estudio tiene como propósito describir la percepción que tienen los estudiantes del INAN sobre su satisfacción con la educación y servicios que reciben en esta casa de estudios, además establecer posibles relaciones entre los elementos que componen la satisfacción del estudiante de educación superior. De esta manera, se llevó a cabo el estudio de caso tomando como fundamento teórico los aportes de Gento y Vivas (2003). El estudio se enmarca en el paradigma cuantitativo, con un diseño no experimental con modalidad de campo. La técnica aplicada fue la encuesta y el instrumento un cuestionario con cinco opciones de respuesta. La población objeto de estudio fue de 109 sujetos pertenecientes a las diferentes carreras que ofrece la institución. El análisis de los datos tanto a nivel descriptivo como inferencial se realizó haciendo uso de una hoja de cálculo Excel del paquete de office y el software SPSS 24. Posteriormente se realizó un análisis inferencial del estudio por medio de una prueba de correlación que permitió establecer la relación o asociación entre las dimensiones del modelo El SEUE. Los hallazgos encontrados revelan que los estudiantes muestran insatisfacción en aspectos referidos a las condiciones básicas de infraestructura y servicios de la institución. Por otro lado, se destacan índices de satisfacción por la formación integral y profesional que reciben en la institución. Finalmente se menciona que el 
estudio permitió identificar relaciones entre diferentes dimensiones que conforman el modelo y la satisfacción por la pertenencia a la institución al grupo de clase.

Palabras claves: estudiantes universitarios, satisfacción estudiantil, educación superior.

\begin{abstract}
The purpose of this study is to describe the perception that INAN students have of their satisfaction with the education and services they receive at this university, as well as to establish possible relationships between the elements that make up higher education student satisfaction. Thus, the case study was carried out taking as a theoretical foundation the contributions of Gento and Vivas (2003). The study is framed within the quantitative paradigm, with a non-experimental design with field modality. The technique applied was the survey and the instrument was a questionnaire with five response options. The study population consisted of 109 subjects belonging to the different careers offered by the institution. The data analysis at both descriptive and inferential levels was carried out using an Excel spreadsheet from the office package and the SPSS 24 software. Subsequently, an inferential analysis of the study was carried out by means of a correlation test that allowed establishing the relationship or association between the dimensions of the SEUE model. The findings reveal that students show dissatisfaction in aspects related to the basic conditions of infrastructure and services of the institution. On the other hand, satisfaction indexes stand out for the integral and professional training they receive at the institution. Finally, it is mentioned that the study made it possible to identify relationships between different dimensions that make up the model and satisfaction with belonging to the institution and the class group.
\end{abstract}

Key words: university students, student satisfaction, higher education.

\title{
1 INTRODUCCIÓN
}

En la actualidad existe un alto interés en nuestro país por mejorar la calidad educativa de los institutos superiores, así bien, lo determina el Plan Nacional de Desarrollo 2017-2021, toda una vida (Senplades, 2017) donde se garantiza potencializar la formación técnica y tecnológica con proyección a futuro de los jóvenes ecuatorianos, brindándoles mejores oportunidades; de esta manera la satisfacción estudiantil es el elemento clave al momento de valorar la calidad de educación ofrecida, en donde se pretende conocer las expectativas que tienen los estudiantes con respecto a los servicios educativos y administrativos (Jiménez, Terriquez, \& Robles, 2011).

La satisfacción de los estudiantes se sostiene en la calidad de vida universitaria y la formación académica que reciben en su casa de estudios, según lo afirman trabajos realizados en diferentes países e instituciones de educación (Joo, Lim, \& Kim, 2011). Para Salinas, Morales, \& Martínez (2008) el eje central de la satisfacción estudiantil es la actividad docente y la condición de las aulas. Estudios realizados por (Caballero, Abello, \& Palacios (2007) señalan que el buen rendimiento académico se correlacionan de manera positiva con la satisfacción que siente el estudiante con su institución educativa; pero en el estudio de Hon, Rasli, \& Chy (2011) se observa lo contrario, puesto que los estudiantes mas satisfechos eran los que obtenían las notas mas bajas en rendimiento académico. 
Casanova (2012) se refiere a la calidad educativa como un proceso de optimización del aprendizaje formativo, el cual depende de actividad docente y de las reformas que se dan en la metodología y las técnicas de enseñanza; así mismo, la instrucción superior se valora mediante las capacidades, asimilación de contenidos y actitudes del alumnado; según esto la calidad educativa es un mejoramiento progresivo con o sin fallos y que debe ser generada como el centro de cualquier organización (Clemenza, Ferrer, \& Pelekais, 2005). Por lo tanto, si la sociedad avanza la educación también debe hacerlo al mismo ritmo, actualizándose e innovando constantemente en vista de los acontecimientos sociales que se producen, además se deben generar iniciativas que permitan a los institutos ofrecer la mejor calidad en educación (Delgado, 2014; Pérez Juste, 2005).

Diferenes autores (Jiménez, Terriquez, \& Robles, 2011; González, Pino, \& Penado, 2017) consideran que la satisfacción resulta de un proceso que se inicia en la persona y termina en él mismo, en donde se hace referencia a un fenómeno esencialmente subjetivo desde su naturaleza hasta la propia medición e interpretación; además la satisfacción hace referencia a la adecuada respuesta a las expectativas, intereses, necesidades y demandas de los destinatarios; siendo la satisfacción uno de los factores relevantes en la calidad de vida universitaria (El Hassan, 2011; Sirgy, Grzeskowiak, Yu, \& Webb, 2010). En el ámbito educativo la satisfacción del estudiante se ve reflejado por diferentes factores que inciden en la formación educativa y las interacciones presentes con el personal docente, sus compañeros, personales administrativos y de servicios, formación académica; asimismo, con los servicios que brinda la institución y la infraestructura como las instalaciones y el equipamiento de la institución, que consiguieran que el estudiante cubra sus expectativas y necesidades de la mejor forma. A su vez, Alves y Raposo (2004) menciona que la satisfacción del alumno en los estudios universitarios es significativa, pues de esto depende la supervivencia de las instituciones, alcanzar el éxito escolar, la permanencia de los estudiantes en la institución, y especialmente la formación de una valoración positiva boca a boca.

Los estudiantes son los principales beneficiarios de la formación académica y de vital importancia al momento de valorar la educación que reciben, asumiendo que su opinión es un referente importante a tener en cuenta; enfatizando que muy pocas veces se permite a los alumnos criticar los procesos en los que están inmersos, en función de sus propias necesidades y el logro de sus experiencias (Gento \& Vivas, 2003). Alumnos más satisfechos con la calidad de educación recibida y su sentimiento de pertenencia desarrollado, favorecen a una difusión positiva de la institución a la que pertenecen (González, Pino, \& Penado, 2017).

En el presente estudio se analiza la satisfacción de los estudiantes del Instituto Tecnológico Integración Andina INAN extensión Girón, mediante la implementación del cuestionario SEUE (Cuestionario para estimar la satisfacción de los estudiantes universitarios con la educación), con lo que 
se pretende analizar las variables que inciden en la satisfacción o insatisfacción del estudiante, con el propósito de obtener indicadores que contribuyan a la toma de decisiones acertada hacia una gestión educativa de calidad.

\section{MÉTODO:}

La ruta metodológica que se siguió para este estudio fue la siguiente. La investigación se enmarca en el paradigma cuantitativo, con un diseño no experimental con modalidad de campo. La técnica aplicada fue la encuesta y el instrumento un cuestionario con cinco opciones de respuesta. La población objeto de estudio fue de 109 sujetos pertenecientes a las diferentes carreras que se ofrecen en la institución, es importante mencionar que el tipo de muestreo fue de tipo censal en atención a la accesibilidad a los sujetos por parte de los investigadores. El análisis de los datos tanto a nivel descriptivo como inferencial se realizó haciendo uso de una hoja de cálculo Excel del paquete de office y el software SPSS 24.

\section{RESULTADOS.}

A continuación se presentarán los resultados del análisis descriptivo, sobre la valoración que tienen los estudiantes sobre su satisfacción con respecto a los servicios educativos que reciben, de acuerdo a las sub dimensiones del modelo planteado por Gento y Vivas (ob. cit).

En estos resultados destacan los siguientes aspectos, respecto a las condiciones básicas de infraestructura los estudiantes se muestran insatisfechos con un índice de 1,96 $\pm 1,13$ donde resaltan aspectos como, espacios reducidos para el descanso 1,66 $\pm 1,26$ y las instalaciones del bar estudiantil 1,25 $\pm 1,03$. En esta dimensión se resaltan aspectos donde los estudiantes manifiestan estar satisfechos como las siguientes, comodidad del mobiliario 2,41 $\pm 1,05$ y los espacios para el estudio $2,54 \pm 1,1$.

En la dimensión referida a los servicios de la institución los estudiantes evidencian insatisfacción con un índice de 1,73 $\pm 1,1$ específicamente con la dotación de la biblioteca (cantidad y vigencia de los libros y revistas) con un índice de 1,25 \pm 1 y la cantidad de alimentos ofrecidos en el bar 1,1 $\pm 1,09$. En cuanto a los indicadores con los cuales se sienten satisfechos se encuentran el servicio recibido en la oficina de registro y control estudiantil (secretaria) 2,54 \pm 1,1 y los procesos de admisión e inscripción al iniciar los diferentes períodos académicos $2,59 \pm 1,1$.

Por su parte, en lo que se refiere a las condiciones de seguridad se presenta un índice de 1,50 \pm 1,05 indicado con estos valores insatisfacción en esta dimensión especialmente con, las instalaciones deportivas y los alrededores de la institución. En esta dimensión se rescata niveles de satisfacción con, el mobiliario (sin aristas peligrosas, materiales rompibles o inflamables) con un índice de 2,23 $\pm 1,1$.

Los resultados de la dimensión satisfacción por su seguridad socio-económica presenta un índice de 1,69 $\pm 1,06$ presentando en términos generales insatisfacción por parte del estudiante, aquí resaltan los 
siguientes indicadores con un índice de 1,33 \pm 1,01 en el servicio del bar y con un índice de 1,41 $\pm 1,27$ la asignación de becas. Por su parte el indicador que muestra satisfacción en los estudiantes en esta dimensión son las actividades académicas que se desarrollan normalmente en la institución 2,07 \pm 0,96.

En la dimensión satisfacción por la seguridad emocional por el trato afectuoso los resultados muestran satisfacción en términos generales por parte de los estudiantes $2,35 \pm 1,14$. Resaltan aquí los siguientes indicadores, el trato recibido por sus compañeros de grupo 3,06 \pm 1 y el trato ofrecido por las autoridades del instituto 2,63 $\pm 1,16$. En los aspectos a trabajar en esta dimensión está, el trato recibido en el personal de la biblioteca $1,57 \pm 1,31$ y el trato recibido de parte del personal del bar 1,69 $\pm 1,1$.

La dimensión satisfacción por la pertenencia a la institución y al grupo de clase los estudiantes están satisfechos $2,67 \pm 1,07$. Aquí se destaca la valoración hacia los compañeros de clase $3 \pm 1,03$ y la consideración y respeto a sus ideas y propuestas en el aula 2,84 \pm 1 , se resalta también la valoración para los docentes de la institución 2,49 $\pm 1,04$. Seguidamente se presentan los resultados de la dimensión satisfacción por el proceso de enseñanza y aprendizaje donde los estudiantes presentan resultados favorables de satisfacción 2,63 $\pm 1,03$ resaltando los siguientes indicadores, la comunicación con los profesores en el aula 2,88 $\pm 1,05$ y la responsabilidad docente en la asistencia diaria a clase 2,78 $\pm 1,06$. De igual forma el contenido de las asignaturas aparece valorado por los estudiantes $2,71 \pm 1,05$.

En lo referente a la satisfacción por los logros personales, esta dimensión muestra un índice de satisfacción por parte de los estudiantes 2,72 $\pm 0,98$, aquí se menciona que uno de los aspectos más valorados por los estudiantes es la formación ciudadana obtenida (valores desarrollados) 2,77 \pm 1 y con un índice de 2,91 \pm 0,9 satisfacción con el desarrollo integral de su persona.

En cuanto a la dimensión satisfacción por el reconocimiento del éxito personal se encuentran índices de satisfacción en los estudiantes 2,48 $\pm 1,09$ se resalta de este elemento de la satisfacción en el estudiante lo siguiente, el reconocimiento que recibe de parte de sus profesores 2,55 $\pm 1,2$ y también el reconocimiento de su éxito por parte de sus compañeros de clase 2,75 $\pm 1,1$. Finalmente se mencionan los resultados de la satisfacción por la autorrealización personal en la cual se evidencian los índices más altos de satisfacción en los estudiantes $2,84 \pm 0,99$, en cuanto a saber que cada día va adquiriendo mayores conocimientos y destrezas 2,94 \pm 1 y la satisfacción que siente por su futuro profesional 3,10 \pm 1 .

Para determinar las relaciones que se pueden establecer entre los elementos que conforman la satisfacción del estudiante en en el Instituto Superior Tecnológico Integración Andina Extensión Girón, se usó la metodología cuantitativa a través de un análisis estadístico inferencial. El nivel para este objetivo de la investigación es correlacional. Se pretende establecer el grado de relación o asociación no causal existente entre las dimensiones que componen el modelo de Gento y Vivas (ob, cit). Primero se midieron 
las dimensiones y luego, mediante pruebas de hipótesis correlaciónales y la aplicación de técnicas estadísticas, se estimó la correlación.

Se trabajó con una matriz de tabulación de los datos obtenidos con la aplicación del instrumento planteado por Gento y Vivas (ob, cit). La cual permitió realizar el análisis descriptivo de los datos recolectados.

El análisis inferencial partió de la construcción de una matriz de doble entrada. Para facilitar el análisis, se procedió discriminar el modelo planteado por Gento y Vivas (ob, cit) sobre la satisfacción del estudiante universitario en sus diferentes dimensiones (satisfacción por las condiciones básicas de Infraestructura, con los servicios de la institución, por las condiciones de seguridad, por su seguridad socio-económica, por la seguridad emocional por el trato afectuoso, por la pertenencia a la institución y al grupo de clase por la aceptación, por el proceso de enseñanza y aprendizaje, por los logros personales, por el reconocimiento del éxito personal y por la autorrealización personal).

Se consideró como dominante la dimensión prevaleciente aquella que agrupara el mayor número de frecuencias de correlaciones significativas y con un índice de correlación muy bueno entre 0,80 y 1 . Lo anterior se establece como criterio de dimensión predominante y se evidencia en el cuadro 2.

Se utilizó la prueba de correlación Rho de Spearman $(\alpha=0,05)$ para evidenciar las relaciones que puedan existir entre el factor satisfacción por la pertenencia a la institución y al grupo de clase, con las dimensiones que componen el modelo de satisfacción del estudiante universitario.

La hipótesis de partida es que la satisfacción por la pertenencia a la institución y al grupo de clase, están relacionados con las dimensiones que completan el modelo (seguridad emocional por el trato afectuoso, proceso de enseñanza aprendizaje, logros personales, reconocimiento del éxito personal y autorrealización personal). Para ello se estableció el siguiente sistema de hipótesis.

\subsection{PLANTEAMIENTO DE LA HIPÓTESIS ESTADÍSTICA:}

La Prueba de correlación partió de las siguientes hipótesis estadísticas:

\subsubsection{Hipótesis Nula}

Ho: $\mathrm{r}$ xy $=0$ (“las dos variables no están correlacionadas significativamente y tienden a 0”)

\subsubsection{Hipótesis Alternativa}

Hi: $r$ xy $\neq 0$ ("la correlación entre las variables no es igual a cero, están correlacionadas significativamente y tiende a 1 ”) 
El planteamiento de la hipótesis nula sugiere que no existe correlación significativa entre los valores obtenidos para la satisfacción por la pertenencia a la institución y al grupo de clase (X1) y los valores de los indicadores asociados al modelo del modelo El SEUE (Y1; Y2; Y3; Y4; Y5); mientras que la hipótesis alternativa plantea que si existen correlaciones significativas entre las variables anteriores. Para el Nivel de Significación se utilizó un a=0.05. La Zona de rechazo se establece que para todo valor de probabilidad igual o menor que 0.05 , se acepta $\mathrm{H} 1$ y se rechaza Ho.

La pertenencia a la institución y al grupo de clase como factor determinante en la satisfacción del estudiante en el Instituto Superior Tecnológico Integración Andina Extensión Girón.

Las correlaciones encontradas constituyen un nivel de análisis más detallado. A partir de allí los resultados evidencian una correlación entre las dimensiones modelo estudiado. En el factor satisfacción por la pertenencia a la institución y al grupo de clases existe una alta correlación con la seguridad emocional y el trato afectuoso (r: 0,875; p: 0,00). Seguidamente se encuentra la correlación entre el factor estudiado y el proceso de enseñanza aprendizaje (r: 0,856; p: 0,00). También existe una alta correlación con los logros personales (r: 0,854; p: 0,00). De igual manera existe correlación el reconocimiento del éxito personal $(\mathrm{r}: 0,815 ; \mathrm{p}: 0,00)$ y finalmente se destaca la correlación con la autorrealización personal del estudiante (r: 0,807; p: 0,00).

Cuadro 1. Factor satisfacción por la pertenencia y al grupo de clases.

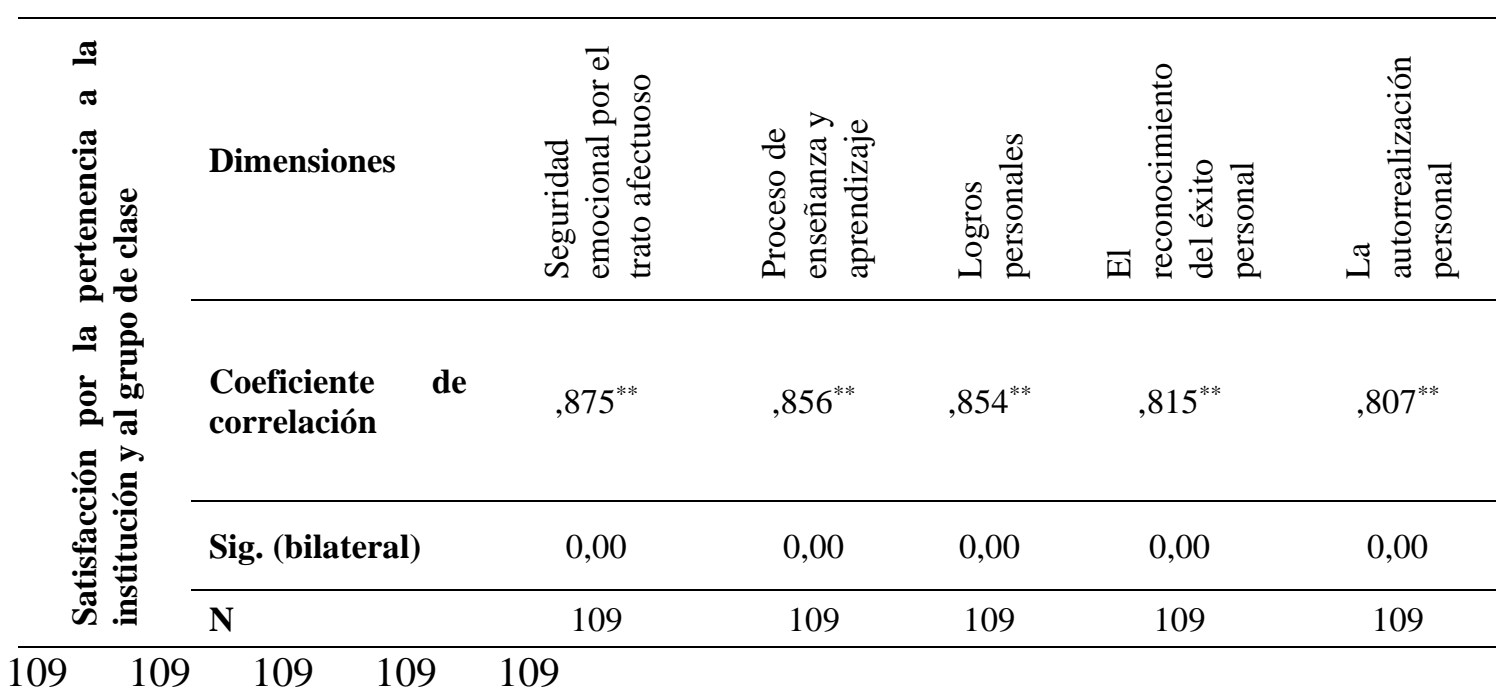

Los hallazgos encontrados revelan que existen evidencias estadísticamente significativas entre las percepciones de los estudiantes y su satisfacción en las dimensiones estudiadas del modelo de Gento y Vivas (ob, cit). Así mismo se establece que la promoción de actividades que promuevan el sentido de pertenencia hacia la institución y al grupo de clase de los estudiantes contribuirá de manera efectiva sobre el resto de dimensiones estudiadas del modelo. 
Estos resultados contribuyen en la definición de estrategias únicas para todos los estudiantes a fin de trabajar en función de la calidad educativa y de la satisfacción del estudiante.

\section{CONSIDERACIONES FINALES.}

El estudio realizado permitió identificar diferentes aspectos relacionados con la satisfacción del estudiante en torno la calidad de educación que reciben en el Instituto Superior Tecnológico Integración Andina INAN extensión Girón. Dentro de lo que se puede destacar se mencionan los siguientes aspectos:

- La mayoría de estudiantes muestran insatisfacción por los aspectos de infraestructura de la institución como son los espacios reducidos para el descanso y las instalaciones del bar estudiantil. Así como también con la dotación de la biblioteca (cantidad y vigencia de los libros y revistas).

- $\quad$ En cuanto a los aspectos referidos a su proceso de formación el estudiante se siente satisfecho con los servicios educativos que recibe en la institución, así como en el trato que recibe por parte de sus compañeros y profesores. También muestra satisfacción en la consideración y respeto a sus ideas y propuestas en el aula.

- Se resaltan los temas referidos a la satisfacción que siente el estudiante en las materias y contenidos que recibe en su formación profesional.

- Los estudiantes en su mayoría se sienten satisfechos con su desarrollo personal e integral con los servicios educativos que reciben y en cuanto a saber que cada día va adquiriendo mayores conocimientos y destrezas en su formación.

- $\quad$ En términos de gestión educativa, el estudio permitió detectar que existen evidencias significativas para realizar la promoción de actividades que promuevan el sentido de pertenencia hacia la institución y al grupo de clase de los estudiantes y que estas contribuirán de manera efectiva sobre el resto de dimensiones estudiadas del modelo. 


\section{BIBLIOGRAFÍA}

Álvarez, J., Chaparro, E., \& Reyes, D. (2015). Estudio de la Satisfacción de los Estudiantes con los Servicios Educativos brindados por Instituciones de Educación Superior del Valle de Toluca. REICE. Revista Iberoamericana sobre Calidad, Eficacia y Cambio en Educación, 13(2), 5-26.

Alves, H., \& Raposo, M. (2004). La medición de la satisfacción en la enseóanza universitaria: El ejemplo de la universidade da beirainterior. International Review on Public and Nonprofit Marketing, 1(1). Obtenido de file:///C:/Users/Mariela/Downloads/EJEMPLO\%201.pdf

Caballero, C. C., Abello, R., \& Palacios, J. (2007). Relación del burnout y el rendimiento académico con la satisfacción frente a los estudios en. Avances en Psicología Latinoamericana, 25(2), 98-111. Obtenido de http://www.redalyc.org/articulo.oa?id=79925207

Casanova, M. A. (2012). El diseño curricular como factor de calidad educativa. Revista Iberoamericana sobre Calidad, Eficacia y Cambio en Educación, 10(4), 15.

Clemenza, C., Ferrer, J., \& Pelekais, C. (2005). La calidad como elemento competitivo en las Universidades. Revista de Artes y Humanidades UNICA, 6(14), 55-83. Obtenido de http://www.redalyc.org/pdf/1701/170118766004.pdf

Delgado, H. B. (2014). El impacto de la calidad educativa. Perú: Universidad Peruana de Ciencias Aplicadas.

El Hassan, K. (2011). Quality of College Life (QCL): Validation of a Measure of Student Well- being in the Middle East. The International Journal of Educational and Psychological Assessment, 8(1), 12-22. Obtenido

de

https://www.researchgate.net/publication/262932109_El_Hassan_K_2011_Quality_of_College_Life_Q CL_Validation_of_a_measure_of_student_well-

being_in_the_Middle_East_International_Journal_of_Educational_and_Psychological_Measurement_8 $1 \_12-22$

Gento, S., \& Vivas, M. (Septiembre de 2003). El SEUE: un instrumento para conocer la satisfacción de los estudiantes universitarios con su educación. Acción pedagógica, 12(2).

González, M., Pino, M., \& Penado, M. (2017). Estudio de la satisfacción percibida por los estudiantes de la UNED con su vida universitaria. RIED. Revista Iberoamericana de Educación a Distancia, 20(1), 243 260.

Hon, T., Rasli, A., \& Chye, L. (2011). Student satisfaction and academic performance: A discriminant analysis approach. Interdisciplinary journal of contemporary research in business, 3(2), 1401-1407.

Jiménez, A., Terriquez, B., \& Robles, F. J. (2011). Evaluación de la satisfacción académica de los estudiantes de la Universidad Autónoma de Nayarit. Revista Fuente Año, 3(6), 46-56.

Joo, J., Lim, K. Y., \& Kim, E. K. (2011). Online university students' satisfaction and persistence: Examining perceived level of presence, usefulness and ease of use as predictors in a structural model. Computers \& Education, 57(2), 1654-1664. doi:10.1016/j.compedu.2011.02.008 
Pérez Juste, R. (2005). Calidad de la educación, calidad en la educación. Hacia su necesaria integración. . Red de Revistas Científicas de América Latina, el Caribe, España y Portugal, 8, 11-33. Obtenido de http://www.redalyc.org/articulo.oa?id=70600801

Salinas, A., Morales, J. A., \& Martínez, P. (2008). Satisfacción del estudiante y calidad Universitaria: un análisis explicatorio en la Unidad Académica Multidisciplinaria Agronomía y Ciencias de la Universidad Autónoma de Tamaulipas, México. Revista de Enseñanza Universitari, 39-55. Obtenido de http://institucional.us.es/revistas/universitaria/31/4SalinasGuti.pdf

Senplades. (2017). Plan Nacional de Desarrollo 2017-2021 Toda una vida. Quito: Secretaría Nacional de Planificación y Desarrollo, Senplades. Obtenido de http://www.planificacion.gob.ec/wpcontent/uploads/downloads/2017/10/PNBV-26-OCT-FINAL_0K.compressed1.pdf

Sirgy, J., Grzeskowiak, S., Yu, G., \& Webb, D. (2010). Quality of College Life (QCL) of Students: Further Validation of a Measure of Well-Being. Social Indicators Research. doi:10.1007 / s11205-010-9587-6 